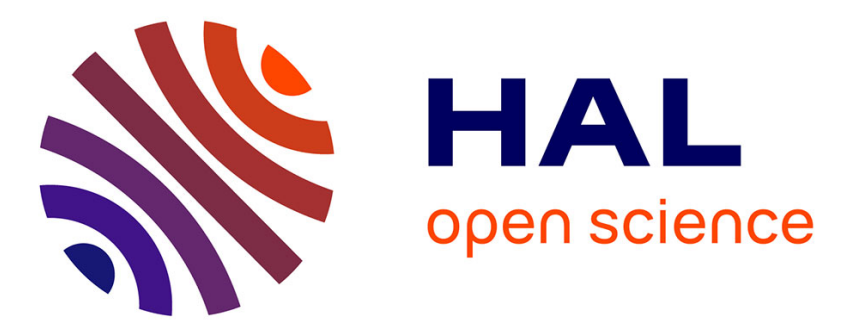

\title{
Bilans de masse du Glacier Blanc entre 1952, 1981 et 2002 obtenus par modèles numériques de terrain
}

\author{
Emmanuel Thibert, Joël Faure, Christian Vincent
}

\section{To cite this version:}

Emmanuel Thibert, Joël Faure, Christian Vincent. Bilans de masse du Glacier Blanc entre 1952, 1981 et 2002 obtenus par modèles numériques de terrain. La Houille Blanche - Revue internationale de l'eau, 2005, 2, pp.72-78. 10.1051/lhb:200502010 . insu-00421361

\section{HAL Id: insu-00421361 \\ https://hal-insu.archives-ouvertes.fr/insu-00421361}

Submitted on 22 Nov 2021

HAL is a multi-disciplinary open access archive for the deposit and dissemination of scientific research documents, whether they are published or not. The documents may come from teaching and research institutions in France or abroad, or from public or private research centers.
L'archive ouverte pluridisciplinaire HAL, est destinée au dépôt et à la diffusion de documents scientifiques de niveau recherche, publiés ou non, émanant des établissements d'enseignement et de recherche français ou étrangers, des laboratoires publics ou privés.

\section{다(1)(2)}

Distributed under a Creative Commons Attribution - ShareAlikel 4.0 International 


\section{La Houille Blanche

\section{Bilans de masse du Glacier Blanc entre 1952, 1981 et 2002 obtenus par modèles numériques de terrain}

\section{Emmanuel Thibert, Joël Faure \& Christian Vincent}

To cite this article: Emmanuel Thibert, Joël Faure \& Christian Vincent (2005) Bilans de masse du Glacier Blanc entre 1952, 1981 et 2002 obtenus par modèles numériques de terrain, La Houille Blanche, 91:2, 72-78, DOI: 10.1051//hb:200502010

To link to this article: https://doi.org/10.1051/lhb:200502010

\section{(c) Société Hydrotechnique de France, 2005}

\section{曲 Published online: 01 Jun 2007.}

\section{Submit your article to this journal $₫$}

\section{Џll Article views: 15}

\section{Q View related articles $\sqsubset$}

Citing articles: 1 View citing articles $\sqsubset 7$ 


\title{
Bilans de masse du Glacier Blanc entre 1952, 1981 et 2002 obtenus par modèles numériques de terrain
}

\author{
Mass balances of the Glacier Blanc between 1952, 1981 and 2002 calculated \\ from digital elevation models
}

Emmanuel Thibert

Parc National des Ecrins, Domaine de Charance, 05000 Gap CEMAGREF-ETNA, BP 76, 38402 Saint Martin d'Hères cedex emmanuel.thibert@cemagref.fr

\section{Joël Faure}

Parc National des Ecrins, Domaine de Charance, 05000 Gap

\section{Christian Vincent}

LGGE, CNRS-UJF, BP 96, 38402 Saint Martin d'Hères cedex

Three Digital Elevation Models (DEM) of the Glacier Blanc have been calculated using photogrammetric restitutions of aerial photographs realised in 1952, 1981 and 2002 by IGN (the french administration for geography) and SINTEGRA (private company, Meylan, France). The aerial triangulation and the orientation of the stereographic images based on GPS control points lead to an absolute orientation whose residual standard deviation is between 0.25 to $1.25 \mathrm{~m}$ in planimetry and between 0.4 and $1.55 \mathrm{~m}$ in altitude (Lambert III, IGN69). Spot heights and 5 meter contour lines have been drawn (120 points/ha) which enables to build a Triangular Irregular Network (TIN) according to Delauney algorithm. This vector network has been linearly interpolated to obtain a DEM which is a raster grid of 10 meter spatial resolution. The subtraction of those DEM yields altitudinal variations on the surface of the glacier from which cumulative mass balance has been deduced. Results show a mass balance increasing of $2.1 \mathrm{~m}$ between 1952 and 1981 and decreasing of $10.8 \mathrm{~m}$ between 1981 and 2002. Results are compared with those of neighbour glaciers.

\section{INTRODUCTION}

\section{I.1 Objectifs}

Les glaciers de montagne sont maintenant largement reconnus comme d'excellents indicateurs des changements climatiques survenus lors des derniers siècles (IPCC, 2001 ; Haeberli, 1995). En particulier, les variations de masse des glaciers peuvent être utilisées pour témoigner du réchauffement du climat au cours du $\mathrm{XX}^{\mathrm{e}}$ siècle (Vincent et al., 2004). Contrairement aux relevés des variations de longueur des glaciers qui sont les données les plus anciennes et les plus répandues (Oerlemans et al., 1998) mais qui fournissent un signal climatique déphasé, filtré (coupure des hautes fréquences) et convo- lué à l'écoulement du glacier (Paterson, 1994 ; Johannesson, 1989), les variations de masse sont des signaux climatiques directs. En effet, la plupart des glaciers des Alpes étant tempérés, ils constituent d'un point de vue thermodynamique des systèmes «ouverts» (pouvant échanger de la matière et de l'énergie) dont la température est au point de fusion. Les échanges d'énergie se manifestent donc directement par une variation de masse (ablation, accumulation interne). Le bilan de masse enregistre également, via l'accumulation, les précipitations de neige de l'hiver. Le suivi du bilan masse permet donc d'accéder aux variations de ces paramètres climatiques. Malheureusement, très peu de glaciers font l'objet d'un tel suivi sur une longue période (Haeberli et al., 1998). Il est néanmoins possible d'accéder au bilan de masse cumulé entre 


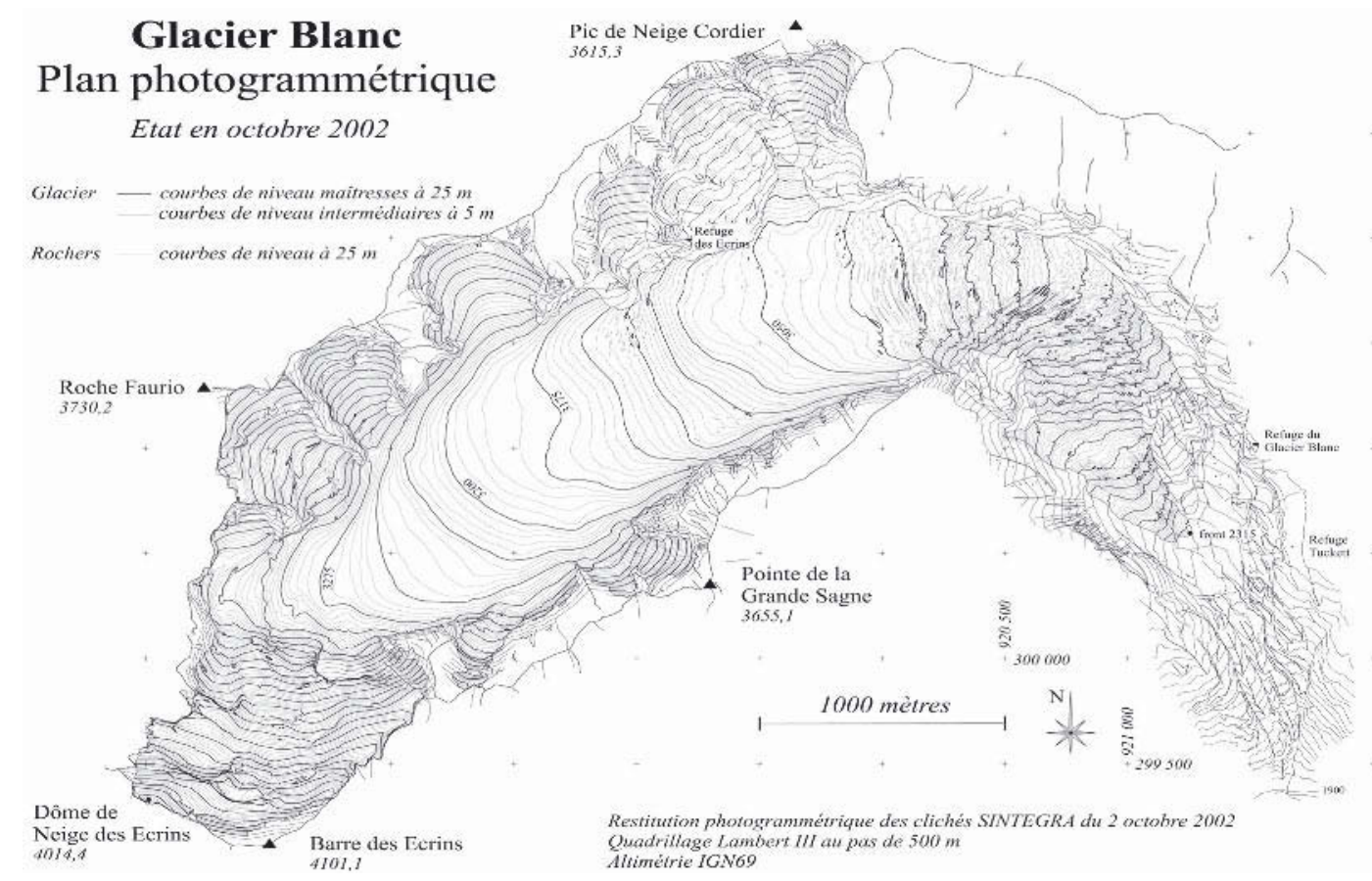

Figure 1 : Plan photogrammétrique du Glacier Blanc dressé à partir de la restitution des clichés SINTEGRA du 2 octobre 2002.

2 dates données en utilisant des documents comme les cartes topographiques et des photographies aériennes.

De tels documents sont disponibles sur le Glacier Blanc pour réaliser un bilan géométrique du glacier et d'en tirer le bilan de masse. Ce glacier $\left(44^{\circ} 56^{\prime} \mathrm{N}, 6^{\circ} 23^{\prime} \mathrm{E}\right.$, Massif des Ecrins, France) est le plus grand glacier du Massif des Ecrins. En octobre 2002, il couvre une surface est de 534 ha, et s'étend sur $5,9 \mathrm{~km}$ de long (distance curviligne). Il prend naissance à $4014 \mathrm{~m}$ au Dôme des Ecrins sur le versant Nord-Est de la Barre des Ecrins (4 $102 \mathrm{~m}$ ), versant dont la pente moyenne est de $30^{\circ}$. Un vaste plateau (pente moyenne inférieure à $10^{\circ}$ ) orienté Est entre 3000 et $3400 \mathrm{~m}$ d'altitude constitue sur 350 ha la plus grande partie du bassin d'accumulation. En dessous de $3000 \mathrm{~m}$, la langue d'ablation orientée Sud-Est (pente moyenne $20^{\circ}$ ) se termine au front à 2315 m d'altitude (fig. 1).

Le Glacier Blanc est par sa taille le plus important glacier méridional de l'Europe de 1'Ouest ayant fait l'objet de suivis plus ou moins réguliers. Les relevés annuels de fluctuations du front les plus anciens remontent à 1889-1907 (Commission Française des Glaciers; Allix 1927). Entre 1921 et 1965, ces relevés sont assurés par le Service RTM de l'Administration des Eaux et Forêts, et sont associés, à partir de 1922, à des mesures de vitesse sur deux profils transversaux situés sur la langue d'ablation (Mougin, 1934; Poncet, 1966). Un troisième profil est installé en amont de la chute de sérac en 1950. Interrompus en 1966, ces relevés de vitesse et de position du front sont repris en 1983 par le laboratoire de Glaciologie (CNRS) jusqu'en 1995 (date où le glacier, en retrait, disparaît du profil aval), puis par le Parc National des Ecrins (Thibert et al., 2003 ; fig. 2). A partir de 1975 et jusqu'en 1991, le Cemagref débute des campagnes périodiques ( 3 ans) de photogrammétrie aériennes qui couvrent uniquement la langue d'ablation du glacier (Burnet et Mura, 1985). En 1968, le bassin supérieur fait

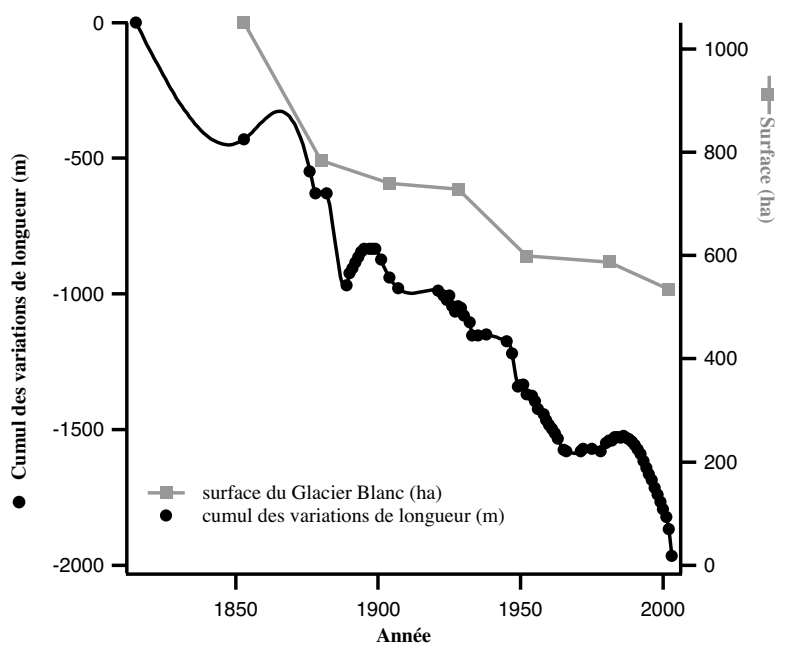

Figure 2: Cumul des variations de longueur et surface du Glacier Blanc entre 1815 et 2003 (d'après Mougin, 1934 ; Letreguilly, 1983 ; Reynaud, 1995 ; Thibert et al., 2003 ; et cette étude).

l'objet d'une campagne de sismique (Gluck, 1969) qui a pour objet de déterminer l'épaisseur de glace sur 2 profils du bassin d'accumulation. Des forages sont également réalisés sur la langue d'ablation pour déterminer quelques épaisseurs (Benoist, 1972). Une synthèse de ces travaux est effectuée par Letréguilly (1983) et Reynaud (1995). Les fluctuations du bilan de masse ont été reconstituées par télédétection sur la période 1985-2000 par Rabatel et al. (2002). Depuis 2000, les bilans de masse sont mesurés dans la zone d'accumulation à l'aide de carottage et de perches d'ablation (Thibert et al., 2003). 
I.2 Principe de calcul du bilan à partir de Modèles Numériques de Terrain (MNT)

Ce calcul du bilan de masse entre 2 états du glacier nécessite la connaissance de l'altitude des points du glacier et également des rives rocheuses qui ont été délaissées et/ou recouvertes entre ces 2 états. La fonction altitude $Z(x, y)$ étant connue sur cette surface $S_{0}$ et exprimée dans un système géoréférencé (coordonnées planimétriques Lambert III et altitude IGN69 dans notre cas), la variation de masse du glacier s'exprimera à partir de la différence $\Delta z$ d'altitude entre les 2 états par la relation :

$$
\Delta m=\iint_{S_{0}} \Delta z(x, y) \rho(x, y) d x d y=\sum_{i} \sum_{j} \Delta h_{i j} \rho_{i j} S_{p i x e l}
$$

où $\rho(x, y)$ désigne la densité moyenne du matériau (glace, névé ou neige) sur l'épaisseur en question. Cette variation de masse peut être également écrite de manière discrète lorsque la fonction altitude n'est connue que sur une grille de points, ce qui sera le cas avec un modèle numérique de terrain qui fournit la matrice $\Delta h_{i j}$ pour chaque pixel de surface $s_{\text {pixel }}$. Dans les deux cas, la variation de masse s'écrit comme le produit de la surface $S_{0}$ par la valeur moyenne du produit de la variation d'altitude par la densité. Le bilan global net entre les deux états est donc donné directement par:

$$
\Delta b=\frac{\Delta m}{S_{0}}=\langle\rho \Delta h\rangle_{S_{0}}
$$

où $\langle\rho \Delta h\rangle_{S_{0}}$ désigne la valeur moyenne sur la surface $S_{0}$ du produit de la densité par la variation d'altitude. Il est important de noter ici que la valeur moyenne $\langle\rho \Delta h\rangle$ ne fournit le bilan net que si la surface recouvre l'ensemble du glacier. Si il ne s'agit que d'une section du glacier, il faut appliquer l'équation de continuité (conservation de la masse) et tenir compte des flux de glace à l'entrée et à la sortie de la section pour relier le bilan à la variation de hauteur moyenne de la section (Reynaud et al., 1986).

\section{II М MÉTHODE}

Les documents disponibles sur le glacier Blanc pour réaliser ce type de travail sont: La carte au 1/10 000 de 1904 de Flusin et al. (1905), la carte du Service Géographique des Armées au 1/20 000 de 1928 (SGA, 1933), puis les couvertures aériennes de l'Institut Géographique National (IGN 1952, 1967, 1981, 1993) et enfin une couverture de SINTEGRA réalisée en 2002 spécialement pour cette étude. En complément de la couverture SINTEGRA de 2002 servant de référence, notre choix s'est porté sur :

- les clichés IGN datant de 1952, première couverture aérienne du massif des Ecrins,

- les clichés IGN de 1981 qui présentent l'intérêt d'être contemporain du maximum de bilan de masse mesuré à cette époque sur les glaciers de Sarennes et Saint Sorlin (Vincent, 2002).

L'extension des prises de vues réalisées par SINTEGRA a été choisie de manière à couvrir les terrains occupés par le glacier Blanc tels qu'ils figurent sur la carte de Flusin et al.

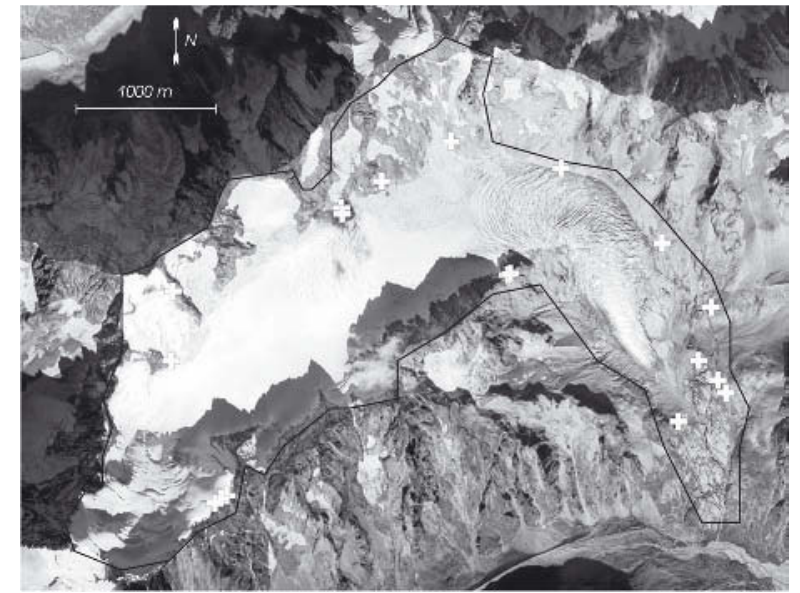

Figure 3 : Orthophotoplan du glacier Blanc obtenu à partir des prises de vues du 2 octobre 2002. Les croix blanches sont les points utilisés pour le calage des 3 couvertures aériennes de 1952, 1981 et 2002 . Le trait noir indique la limite des terrains restitués pour obtenir le modèle numérique de terrain de référence.

(1905) et de pouvoir ainsi calculer ultérieurement un bilan de masse à partir de ce document (fig. 3).

\section{II.1 Paramètres des prises de vue}

Les clichés de 1952 proviennent de la mission La Grave - Chorges F3435-3438 du $1^{\text {er }}$ août 1952, soit 6 clichés sur deux axes de vol, échelle au centre 1/25 000. Les clichés de 1981 proviennent de la mission IFN05 P (inventaire forestier national, département des Hautes Alpes) du 29 juillet 1981. Deux axes de vol couvrent le glacier en 8 clichés dont l'échelle au centre est d'environ 1/17 000. La couverture SINTEGRA a été effectuée en fin de période d'ablation, le 3 octobre 2002 sur un axe de vol comportant 5 clichés au $1 / 20000$.

\section{II.2 Stéréopréparation}

La campagne de stéréopréparation a été effectuée en août 2001. Compte tenu de l'antériorité des prises de vues IGN, des points remarquable du relief (gendarmes, blocs de rochers, toiture des refuges) ont été choisis d'après leur visibilité sur les anciens clichés. En complément, des points ont été implantés (croix de peinture blanche et bâches) en prévision de la couverture aérienne SINTEGRA de 2002. Dix-huit points d'appui ont été utilisés (fig. 3). Ils ont été rattachés au système planimétrique NTF — Lambert III et altimétrique NGF - IGN69 par géodésie GPS différentiel bi-fréquence (statique rapide post-traité). La précision est de l'ordre de quelques $\mathrm{cm}$ en planimétrie et d'une dizaine de centimètres en altimétrie relativement aux points géodésiques utilisés (IGN, réseau REGAL).

\section{II.3 Calage des clichés}

Ce travail ainsi que la restitution ont été réalisés par SINTEGRA sur un restituteur de type analytique (stéréorestituteur $\mathrm{M} / \mathrm{s}$ LEICA DSR 15). Les couvertures étant 
Tableau 1. Erreur de calage des clichés $\sigma_{x y}$ (planimétrique) et $\sigma_{z}$ (altimétrique), erreur altimétrique résultante $\Delta Z$ pour le MNT par rapport aux points d'appui, incertitude sur les variations d'altitude $\Delta H$ entre le MNT $(1952,1981)$

et le MNT de référence (2002) et incertitude résultante $\Delta b$ sur le bilan de masse calculé par rapport à l'année 2002.

\begin{tabular}{|c|c|c|c|c|c|}
\hline Année/précision & $\sigma_{\mathbf{x y}}$ & $\sigma_{\mathbf{z}}$ & $\Delta \mathbf{Z}$ & $\Delta \mathbf{H}$ & $\Delta \mathbf{b}$ \\
\hline 1952 & $1,25 \mathrm{~m}$ & $1,55 \mathrm{~m}$ & $2,0 \mathrm{~m}$ & $2,1 \mathrm{~m}$ & $1,9 \mathrm{~m}$ eq. eau \\
\hline 1981 & $0,25 \mathrm{~m}$ & $0,40 \mathrm{~m}$ & $0,50 \mathrm{~m}$ & $0,96 \mathrm{~m}$ & $0,9 \mathrm{~m}$ eq. eau \\
\hline 2002 & $0,45 \mathrm{~m}$ & $0,55 \mathrm{~m}$ & $0,72 \mathrm{~m}$ & - & - \\
\hline
\end{tabular}

parfois constituées de plusieurs couples stéréo sur 2 axes de vol, et le nombre de points d'appui étant réduit, il a d'abord été réalisé une aérotriangulation (calages des clichés entre eux) puis un calage de cet ensemble par rapport au réseau géodésique (calcul en bloc par un programme de compensation type moindres carrés). La précision en planimétrie et altimétrie $\left(\sigma_{\mathbf{x y}}, \sigma_{\mathbf{z}}\right)$ de l'orientation absolue des clichés ainsi obtenue est indiquée au tableau 1.

\section{II.4 Restitution photogrammétrique}

Afin d'établir les Modèles Numériques de Terrain (MNT) du glacier, les courbes de niveau ont été filées tous les 5 mètres (sur le glacier, les rives rocheuses et les terrains déglacés depuis 1904). Des détails bien visibles par l'opérateur dans le stéréomodèle ont été également pointés pour fournir des points côtés entre les courbes de niveau (certaines crevasses et séracs, gendarmes, crêtes, talwegs et blocs rocheux). Avec la densité de points obtenue (environ 120 points/ha), il est alors possible d'obtenir un plan photogrammétrique au 1/5000 et un modèle numérique de terrain jusqu'à 2,5 mètres de résolution spatiale. La figure 1 illustre le plan obtenu à partir des clichés d'octobre 2002. L'orthophotoplan issu des mêmes clichés est représenté à la figure 3 .

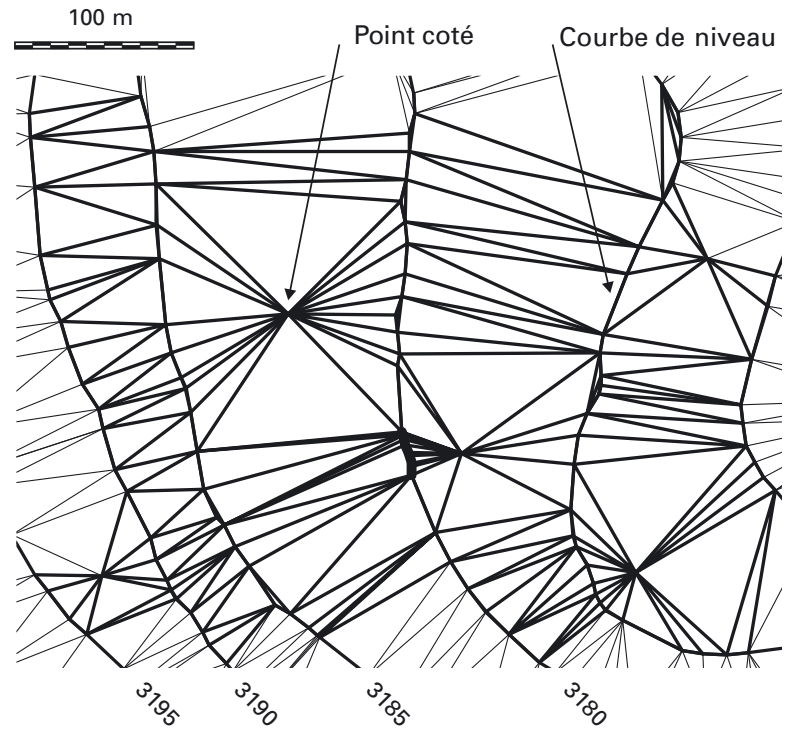

Figure 4 : Réseau de triangle irrégulier (TIN) construit sur les courbes de niveau et les points côtés. Aucun côté de triangle ne coupe les courbes de niveau.

\section{II.5 Modèle numérique de terrain}

Le MNT vecteur construit est de type réseau de triangle irrégulier (Triangular Irregular Network $=\mathrm{TIN}$ ) s'appuyant sur les angles des courbes de niveau et les points côtés (cf. fig. 4). L'algorithme retenu pour construire ce réseau est celui de Delauney (les angles des triangles sont maximisés). Les algorithmes classiques de triangulation spécifiques aux courbes de niveau (triangulation contrainte) n'ont pas été retenus pour pouvoir inclure les points côtés dans ce réseau. Néanmoins, la densité des courbes de niveau est telle que tous les segments de courbes de niveau constituent le côté d'un triangle. Par conséquent, aucune courbe de niveau n'est coupée par le côté d'un triangle. Ceci assure la continuité entre les altitudes fournies par les courbes de niveau et le réseau de triangle. Le résultat du calcul de triangulation a donc de ce point de vue la même propriété que celui des algorithmes de triangulation contraints et incorpore en plus les points côtés.

Le réseau de triangle ainsi obtenu a été interpolé sur une grille de $10 \mathrm{~m}$ (quadrillage Lambert III) pour obtenir le modèle numérique d'altimétrie $(530 \times 420$ pixels $)$. L'interpolation est linéaire: 1'altitude de chaque pixel est celle du point du triangle intercepté par le centre du pixel. Ce MNT est donc un fichier de type image (raster) dont l'attribut est l'altitude (IGN 69). La soustraction des MNT donne les variations d'altitude et donc le bilan de volume

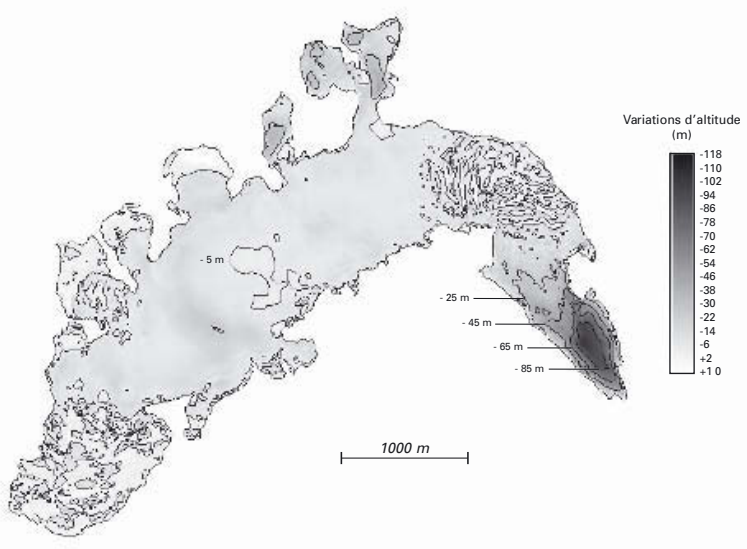

Figure 5 : Carte des variations d'altitude du glacier entre 1952 et 2002 représenté en 256 niveaux de gris. En noir figurent les courbes d'isovaleur des pertes d'altitudes à $20 \mathrm{~m}$ (entre -5 et $-85 \mathrm{~m}$ ). La perte moyenne d'altitude du glacier est de 11,2 mètres. 


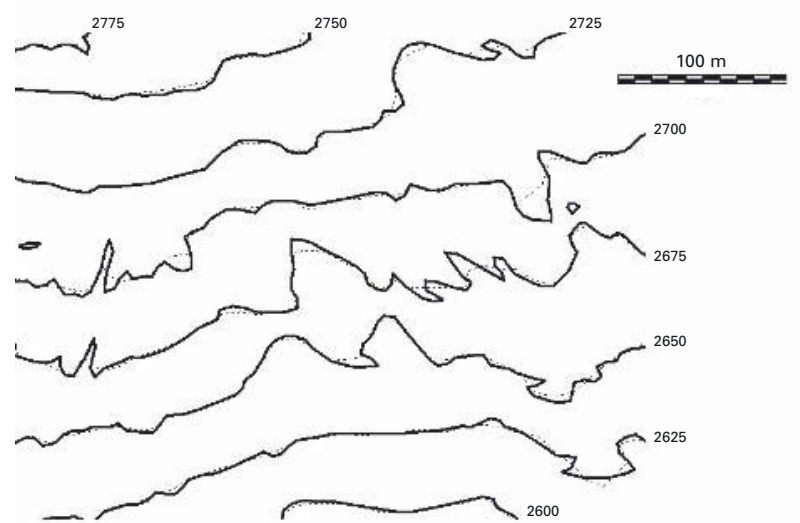

Figure 6 : Tracé des courbes de niveau obtenues par le stéréorestituteur (en trait noir continu) et par le modèle numérique de terrain à $10 \mathrm{~m}$ de résolution (en pointillé).

Dans les zones au relief complexe, comme ici dans

la chute de séracs, les courbes de niveau du MNT ont tendance à simplifier le relief à petite échelle (en dessous de $20 \mathrm{~m}$ ) en supprimant les crêtes et talwegs.

entre deux états du glacier. La figure 5 représente les variations d'altitude entre 1952 et 2002 et les courbes d'isovaleur de variation d'altitude à $20 \mathrm{~m}$.

\section{RÉSULTATS}

\section{III.1 Fidélité du MNT au plan photogrammétrique}

Elle a été évaluée en comparant les courbes de niveau calculées d'après le MNT à celles obtenues directement par le stéréo-restituteur. Les discordances de tracé sont inférieures à $1 \mathrm{~m}$ dans la plupart des cas. Seul dans les zones où le relief est complexe à petite échelle (en dessous de $20 \mathrm{~m}$ ), le maillage d'interpolation du MNT $(10 \mathrm{~m})$ moyenne le relief. Les courbes de niveau du MNT ont un rayon de courbure qui est plus élevé que celui des courbes données par le stéréo-restituteur (fig. 6). Les crêtes et talwegs de petite échelle $(<20 \mathrm{~m})$ ont tendance à être éliminés. La densité de point permettrait d'augmenter la résolution du MNT jusqu'à un mètre dans ces zones ou d'adopter une grille à pas variable. Cependant, l'information recherchée étant le bilan global net sur l'ensemble du glacier, nous avons conservé le maillage à 10 mètres.

\section{III.2 Incertitude sur les altitudes}

L'erreur globale $\Delta Z$ associée à l'altitude fournie par le MNT $Z(x, y)$ provient a) de l'erreur de calage altimétrique des clichés, b) de l'erreur de calage planimétrique qui induit dans les zones à relief une erreur d'altitude du premier ordre et c) de la résolution de la grille du MNT. Cette dernière erreur semble négligeable compte tenue de la bonne fidélité au plan photogrammétrique du MNT à $10 \mathrm{~m}$. Les deux premières contributions peuvent s'écrire :

$$
\Delta Z=(\Delta z)_{\text {calage }}+\left(\frac{\partial Z}{\partial x}\right)_{y} \Delta x+\left(\frac{\partial Z}{\partial y}\right)_{x} \Delta y=\sigma_{z}+\sigma_{x y} \operatorname{grad} Z
$$

où $\sigma_{z}$ désigne l'erreur de calage altimétrique des clichés, $\sigma_{x y}$ l'erreur de calage planimétrique et gradZ le gradient moyen du MNT sur le glacier. Avec un gradient moyen de $38 \%$ pour les trois années étudiées, cette erreur peut se calculer en fonction des erreurs de calage de chaque ensemble de clichés. Il en résulte l'erreur $\Delta H$ sur les variations d'altitude entre les MNT de 1952 et 1981 par rapport au MNT de référence de 2002, et l'erreur résultante $\Delta b$ sur le bilan de masse (tab. 1).

\section{III.3 Calcul du bilan de masse}

La différence d'altitude donnée par les MNT doit être convertie en lame d'eau moyennant une hypothèse sur la densité. On a ici simplement pris comme facteur 0,9 sur l'ensemble du glacier pour passer à l'équivalent en eau.

Nous avons tenu compte des dates précoces des prises de vue de l'IGN (1 $1^{\text {er }}$ août 1952 et 29 juillet 1981) pour faire une correction de lame d'eau correspondant à l'ablation jusqu'à début octobre, mois de la prise de vue des clichés de référence de 2002. Pour ceci, nous avons calculé le cumul de température positive sur août et septembre (degrés jours positifs) pour chaque point du glacier en utilisant les normales saisonnières de la station MétéoFrance de Pelvoux les Claux (1 $270 \mathrm{~m})$.

Le MNT fournissant l'altitude, le cumul de température est calculé en utilisant le gradient adiabatique de $5,510^{-3}{ }^{\circ} \mathrm{C} / \mathrm{m}$. Nous utilisons 2 facteurs de sensibilité correspondant au matériau glace en dessous de $3000 \mathrm{~m}\left(0,7 \mathrm{~cm} /{ }^{\circ} \mathrm{C} /\right.$ jour, Torinesi et $a l ., 2001)$ et au névé au dessus de $3000 \mathrm{~m}\left(0,5 \mathrm{~cm} /{ }^{\circ} \mathrm{C} /\right.$ jour, Thibert et al,. 2003). Cette correction donne à titre d'exemple environ $-3,8 \mathrm{~m}$ d'eau à $2250 \mathrm{~m}$ et $-1,2 \mathrm{~m}$ d'eau à $3100 \mathrm{~m}$ pour la période août-septembre. La lame d'eau de fonte pondérée sur l'ensemble du glacier est de $-1,4 \mathrm{~m}$ pour les deux années 1952 et 1981 . Les résultats sont rassemblés au tableau 2.

Tableau 2. Bilan volumique et bilan de masse du glacier Blanc pour les périodes 1952-2002 et 1981-2002.

\begin{tabular}{|c|c|c|c|c|}
\hline Période & $\begin{array}{c}\text { Bilan volumique } \\
(\mathbf{m})\end{array}$ & $\begin{array}{c}\text { Bilan de masse } \\
(\mathbf{m} \text { eq. Eau })\end{array}$ & $\begin{array}{c}\text { Correction d'ablation } \\
(\mathbf{m} \text { eq. Eau) }\end{array}$ & $\begin{array}{c}\text { bilan corrigé } \\
\text { (m eq. Eau) }\end{array}$ \\
\hline $1952-2002$ & $-11,2$ & $-10,1$ & 1,4 & $-8,7+/-1,9$ \\
\hline $1981-2002$ & $-13,5$ & $-12,2$ & 1,4 & $-10,8+/-0,9$ \\
\hline
\end{tabular}




\section{DISCUSSION}

En terme de variation du stock d'eau, ces chiffres correspondent à une perte de 52 millions de $\mathrm{m}^{3}$ d'eau entre 1952 et 2002 . Le glacier blanc a regagné 12,4 millions de $\mathrm{m}^{3}$ entre 1952 et 1981 . La perte de volume d'eau se chiffre à 63,4 millions de $\mathrm{m}^{3}$ entre 1981 et 2002 . La surface du glacier de 599 ha en 1952, s'abaisse à 587 ha en 1981 (alors que son volume a augmenté), et passe à 534 ha en 2002 .

Les résultats obtenus pour le glacier Blanc sont comparés aux mesures de bilan cumulé et de bilan obtenu également par photogrammétrie sur les glaciers de Sarennes, St Sorlin et Argentière (tab. 3 et fig. 7, d'après Vincent, 2002 et Michel et al., 2003).

Sur la période 1981-2002, le bilan moyen annuel est de $-0,51 \mathrm{~m}$ eq. eau/an au glacier Blanc, identique à celui du glacier d'Argentière, mais environ deux fois moins élevé qu'au glacier de Sarennes ( $-0,98 \mathrm{~m}$ eq. eau/an), le glacier de St Sorlin ayant un comportement intermédiaire avec $-0,77 \mathrm{~m}$ eq. eau/an.

Sur la période 1952-1981, le glacier Blanc voit son bilan augmenter de $2,1 \mathrm{~m}$ eq. eau. Les bilans sont également positifs pour Argentière ( $+5,9$ m eq. eau) et St Sorlin $(+0,4 \mathrm{~m}$ eq. eau), alors que Sarennes est en déficit $(-10,9 \mathrm{~m}$ eq. eau).

Malgré sa position méridionale, le glacier Blanc semble avoir un comportement proche de celui du glacier d'Argentière. Plusieurs éléments peuvent être avancés pour expliquer cette similitude : tout comme Argentière, le glacier Blanc est un glacier de grande taille ( 534 ha et $5,9 \mathrm{~km}$ de long en 2002) disposant d'un vaste bassin d'accumulation à haute altitude $(80 \%$ de la surface est entre 3000 et $4000 \mathrm{~m})$. Ce bassin est situé sous les plus hauts sommets du massif et l'effet orographique doit probablement forcer la pluviométrie. Les mesures d'accumulation effectuées sur le bassin indiquent en effet, à $3100 \mathrm{~m}$, deux fois et demi plus de précipitation que ce qui est mesuré par MétéoFrance à la station de Pelvoux les Claux à $1270 \mathrm{~m}$ (Thibert et al., 2003). Ainsi, les glaciers qui ont des zones d'accumulation élevées sont beaucoup moins sensibles aux variations du bilan d'énergie estival. Et, ce sont d'ailleurs principalement ces conditions estivales qui ont affecté les glaciers alpins au cours du $\mathrm{XX}^{\mathrm{e}}$ siècle et, en particulier, au cours des 20 dernières années (Vincent et al., 2004). Une autre hypothèse pouvant expliquer une plus faible variabilité du bilan serait l'existence d'une zone d'accumulation froide dans la partie supérieure de la face Nord Est des Ecrins. Son réchauffement aurait alors absorbé une partie de l'énergie qui n'aurait pas contribué à la fusion de la glace. Mais cette hypothèse n'est absolument pas vérifiée pour l'instant.

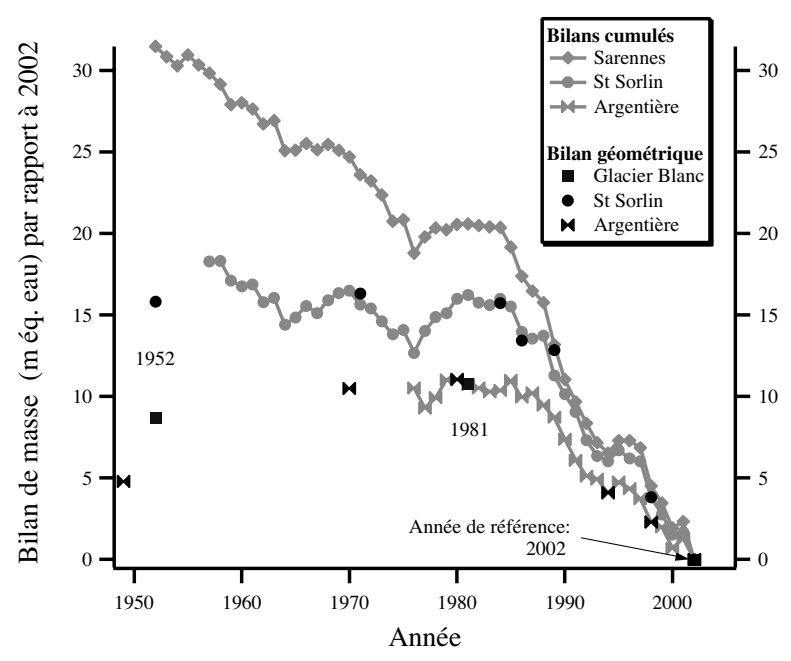

Figure 7 : Comparaison sur la période 1952-2002 des bilans de masse cumulés obtenus à partir des mesures de terrain (méthode glaciologique : glaciers de Sarennes, St Sorlin et Argentière) et par photogrammétrie (glaciers Blanc, St Sorlin et Argentière). Données d'après Vincent (2002), Michel et al. (2003) et cette étude.

Tableau 3: Comparaison des bilans de masse mesurés à Sarennes, St Sorlin, Argentière et glacier Blanc.

\begin{tabular}{|c|c|c|c|c|}
\hline Période/bilan (m eq. eau) & Sarennes & St Sorlin & Glacier Blanc & Argentière \\
\hline $1952-2002$ & $-31,5$ & $-15,8$ & $-8,7+/-1,9$ & $-4,8(1949)$ \\
\hline $1952-1981$ & $-10,9$ & $+0,4$ & $+2,1+/-2,1$ & $+5,9(1949)$ \\
\hline $1981-2002$ & $-20,6$ & $-16,2$ & $-10,8+/-0,9$ & $-10,7$ \\
\hline
\end{tabular}

\section{REMERCIEMENTS}

Les auteurs tiennent à remercier R. Chevalier et C. Roger pour leur aide lors de la campagne de stéréopréparation, ainsi que B. Barré et I. Prévitali de la société SINTEGRA pour la qualité des travaux de restitution. Cette étude a été financée par le Parc National des Ecrins (Ministère de l'Ecologie et du Développement Durable).

\section{BIBLIOGRAPHIE}

Allix A., (1927). — Mémoire sur les observations glaciologiques faites en Dauphiné jusqu'en 1924. Etudes glaciologiques, Tome VI, p. 1-138. Imprimerie Nationale, Paris.

Benoist J.-P. (1972). — Déformation d'une langue glaciaire (Glacier Blanc), thèse de troisième cycle, Université de Grenoble, publication $\mathrm{n}^{\circ} 162$ du laboratoire de glaciologie du CNRS, $69 \mathrm{p}$. 
Burnet R. et Murat R. (1985). - Surveillance des glaciers par photogrammétrie aérienne, Cemagref, division nivologie, Grenoble, 6 p.

Flusin G., JaCOB C. (1905). - Carte des glaciers Blanc et Noir (levés de terrain de 1904).

GLuCK S. (1969). - Epaisseur du glacier Blanc (Massif de l'Oisans) dans sa partie supérieure. Comptes Rendus de l'Académie des Sciences, Série D, n² 268, p. 1583-1585.

Haeberli W. (1995). - Glacier fluctuations and climate change detection. Geogr. Fis. Dinam. Quat., 18, 191-199.

Haeberli W., Hoelzle M., Suter S. \& Frauenfelder R. (1998). Fluctuation of glaciers, 1990-1995, Vol. VII, UNESCO, Paris.

Institut GÉOGRAPhiQue National (1952). - Mission La GraveChorges, (1967) Mission F XXIV-36. (1981) Mission IFN 05 P. (1993) Mission FD 05

Intergouvernemental PAnel on Climate Change (IPCC) (2001). - Climate Change 2001, Contribution of Working Group I to the Third Assessement Report of the Intergouvernemental Panel on Climate Change, Cambridge Univ. Press, New York.

Johannesson T., RAYMOnd C. \& Waddington E. (1989). - Timescale for adjustment of glaciers to changes in mass balance. J. Glaciolo., 35, 355-369.

LetréGuilly A. (1983). - Etude des mesures effectuées sur le glacier Blanc (Massif des Ecrins), Mémoire de DEA, Laboratoire de Glaciologie (CNRS), Université Joseph Fourier, Grenoble.

Michel J.-P., Gay M. et Valla F. (2003). - Bilan de masse du glacier de Sarennes, $54^{\mathrm{e}}$ saison 2001-2002, SHF, Grenoble.

Mougin M. (1934). - Glacier du Pelvoux et Vénéon, p. 71-88; Variations du volume des langues terminales: p. 203-210; Cinématique glaciaire: p. 252-255, Observations sur la neige et les glaciers des massifs méridionaux du Pelvoux: p. 272-290. Etudes glaciologiques, Paris, Imprimerie Nationale, Tome VII
Oerlemans J. et al. (1998). - Modelling the response of glaciers to climate warming. Clim. Dyn., 14, 267-274.

PAterson W.S.B. (1994). - The physics of glaciers, $3^{\text {rd }}$ ed., Elsevier Sci., New York.

Poncet A. (1966). - Observations glaciologiques sur les glaciers Blanc et Noir. Centre technique forestier de Grenoble, 20 décembre 1966, $13 \mathrm{p}$.

Rabatel A., J.-P. Dedieu, et L., Reynaud (2002). — Reconstitution des fluctuations du bilan de masse du Glacier Blancs (Massif des Écrins, France) entre 1987 et 2000 par télédétection optique (imagerie Spot et Landsat). La Houille Blanche, $n^{\circ} 6 / 7$, p 64.

Reynaud L., Vallon M. et Letreguilly A. (1986). - Mass balance measurements : problems and two new methods of determining variations. Journal of Glaciology, Vol. 32, $\mathrm{n}^{\circ} 112$.

REYNAUD L. (1995). — Rapport d'étude sur les suivis glaciologiques dans le Parc National des Ecrins, Laboratoire de Glaciologie et de Géophysique de l'Environnement, Parc National des Ecrins, $11 \mathrm{p}$.

Service GéographiQue de L'Armée (1933). - Carte 1/20 000, 3436, Saint-Christophe en Oisans, feuilles 3 et 4.

Thibert E., FaureJ., Vincent C. et Reynaud L. (2003). Monitoring of glacier Blanc, french Alps. Alpine Glaciological Meeting, Grenoble, France.

Torinesi O., Letréguilly A. \& Valla F. (2001). - A century reconstruction of the mass balance of Glacier de Sarennes, French Alps. Journal of Glaciology, Vol. 48, 142-148.

VINCENT C. (2002). - Influence of climate change over $20^{\text {th }}$ century on four french glacier mass balances. Journal of Geophysical Research, Vol. 107, No. D19, 4375.

Vincent C., Kappenberger G., Valla F., Bauder A., Funk M. \& Le Meur E. (2004). - Ice ablation as evidence of climate change in the Alps over the $20^{\text {th }}$ century. Journal of Geophysical Research, Vol. 109, No. D10, 10104. 


\section{SOCIETE HYDROTECHNIQUE DE FRANCE}

25 rue des Favorites - F 75015 PARIS - Tél. 01-42-50-91-03 - Fax 01-42-50-59-83 - mail shf@shf.asso.fr - www.shf.asso.fr SIRET 78430905600025 - Code APE 731Z - Code TVA FR23784309056 - CCF PARIS VAUGIRARD 30056-00073-00735402180-23 - CCP Paris : 6148 20 N

COLLECTION : EAU ET ENVIRONNEMENT

Crues et Laves torrentielles (1992)

La pluie, source de vie, choc de pollution (1993)

Glaciologie et nivologie - Etat des recherches et des connaissances à la fin du XXè s.(1995)

Le risque crue en région parisienne (1997)

La gestion des risques liés aux inondations (1999)

Protection des eaux souterraines (mai 2000)

Gestion des sédiments, de la source à la mer (mars 2001)

Imagerie satellitaire et radar, au service de l'eau (juin 2001)

Forêts et eau (septembre 2001)

Rencontres de Grenoble (novembre 2001)

Variations climatiques et Hydrologie (décembre 2001)

Hidroenergia 2002 - Conférence sur la Petite Hydroélectricité (juillet 2002)

Le SAGE, une chance pour la politique de l'eau, (mai 2003)

Quels modèles physiques pour le 21è siècle ? (octobre 2003)

Etiages et crues extrêmes régionaux en Europe : perspectives historiques (janvier 2004)

Crues méditerrannéennes : vulnérabilité, aléa, prévision et gestion de crise (juin 2004)

Euros Nb

75,00

30.00

30.00

60,00

75,00

75,00

75,00

75,00

75,00

30.00

75,00

100.00

55,00

60,00

60,00

60,00

\section{COLLECTION : MECANIQUE DES FLUIDES}

3ème journées Cavitation (1996)

Hydrodynamique des procédés industriels diphasiques (1998)

Interactions mécaniques entre fluides et structures (novembre 1999)

Microfluidique - microécoulements liquides et gazeux et leurs applications (décembre 2002)

Modélisation des écoulements diphasiques (novembre 2004) - CDrom

Microfluidique - $\mu$ Flu'04 (décembre 2004) - CDRom

75,00

60,00

60,00

90,00

40,00

40,00

\section{COLLECTION : JOURNEES DE L'HYDRAULIQUE}

13èmes. Influence des activités de l'homme sur le cycle hydrométéorologique (1974)

14èmes. La mécanique des Fluides et l'environnement (1976)

15èmes. L'Hydrotechnique au service d'une politique de l'Eau (1978)

16èmes. Rejets de chaleur à l'atmosphère (1980)

17èmes. L'assainissement de demain (1982)

18èmes. L'Hydraulique et la maîtrise du Littoral (1984)

20èmes. Machines hydrauliques (1989)

21 èmes. Les Eaux souterraines et la gestion des eaux (1991)

22èmes. L'Avenir de l'Eau (1992)

23èmes. Crues et Inondations (1994)

24èmes L'Eau, l'Homme et la Nature (1996)

25èmes L'école française de l'eau au service du développement mondial (1998)

26èmes Les logiciels de mécanique des fluides au service de l'industrie et de l'environnement (2000)

27èmes Eau et Economie (2002)

28èmes L'Eau et le Monde vivant (2004)

45,00

45,00

45,00

45,00

épuisé

45,00

45,00

45,00

45,00

épuisé

épuisé

45,00

30.00

45,00

45,00

COLLECTION SHF - ADEME : Petite Hydroélectricité

Guide général (1999)

Intégration dans l'environnement (1998)

Spécifications techniques propres aux Equipements (1997)

Actualité d'une énergie renouvelable (actes du colloque, 1998)

\section{CAHIERS DES CHARGES - TYPES :}

Pour la fourniture de turbines hydrauliques : (édition française ou édition anglaise)

1-turbines Francis, Pelton et Kaplan, + spécifications propres aux turbines Francis (1972)

2-spécifications propres aux turbines Pelton (1972)

3-spécifications propres aux turbines Kaplan (1973)

4-groupes du type Bulbe (1974)

Pour la fourniture et le montage des conduites forcées en acier et de leurs accessoires (1972)

Données techniques annexes (1968)

\section{COLLECTION : HISTOIRE ET HOUILLE BLANCHE}

Actes du 2ème congrès de la Houille Blanche (1914)

Actes du 3ème congrès de la Houille Blanche (1925)

T.V.A. 5,5\% incluse pour la France (Frais de port en sus) 


\section{Nouvelles de l'AIRH : sommaires du Journal of Hydraulic Research}

\section{JHR 2005 VOL 43(2)}

- Decision support model for operation of multi-purpose water resources systems, $O$. $R$. DOLLING and E A. VARAS

- Elaboration of management tool of a reservoir dam on the Sebou river (Morocco) using an implicit hydraulic model, $M$. IGOUZAL and A. MASLOUHI

- Sediment transport capacity in rivers, S. YANG

- Energy losses and threshold conditions for choking in channel contractions, B. WU and A. MOLINAS

- Investigation of near wall velocity in 3-D smooth channel flows, S. Yang, SIOW-YONG and J.A. MCCORQUODALE

- Curvilinear turbulence modelling of open channel flow, J.J.R. WILLIAMS

- Correlation and distribution of shear stress for turbulent flow in a smooth rectangular open channel, A. BILGIL

- Floating breakwaters under regular and irregular wave forcing: reflection and transmission characteristics, $E$. KOUTANDOS, P. PRINOS and $X$. GIRONELLA

- Fall velocities of natural sediment particles: a simple mathematical presentation of the fall velocity law, K. SHE, L. TRIM and D. POPE

- Inverse modeling of unsaturated heterogeneous media by two-phase pressure derivative matching, $\mathrm{K}$. MASUMOTO

- Multi-level fracture network model and FE solution for ground water flow in rock mass, C. JUNRUI, L. SHOUYI and WUYANQING
JHR ISSUE Vol. 43 (3) 2005

- Du Boys and sediment transport, W.H. HAGER

- Physical modelling of the flow field in an undular tidal bore, $\mathrm{H}$. CHANSON MIAHR

- Influence of bed roughness on sediment suspension: experimental and theoretical studies, B.S. MAZUMDER, K. GHOSHAL and D.C. DALAL

- Velocity measurements in a developed open channel flow in the presence of an upstream perturbation, RAM BALACHANDAR and V.C. PATEL

- Success of booster chlorination for water supply networks with genetic algorithms, OSMAN N. OZDEMIR and M. ERKAN UÇANER

- Turbulence particle models for tracking free surfaces, SONGDONG SHAO and HITOSHI GOTOH

- Three-dimensional numerical simulation of wind-driven flows in closed channels and basins, FRANCESCO CIOFFI, FRANCESCO GALLERANO and ENRICO NAPOLI

- Modelling suspended sediment transport using an integrated numerical and ANNs model, $B$. LIN and M.M. NAMIN

- Parameter identification using optimization techniques in openchannel inverse problems, HÉLĖNE ROUX and DENIS DARTUS

- Theoretical and experimental study on flow distribution at fracture intersections, HUYUNJIN, MAO GENHAI, CHENG WEIPING and ZHANG JUNJUN
JHR ISSUE Vol. 43 (4) 2005

- Finite volume modeling of variable density shallow-water flow equations for a well-mixed estuary: application to the Río Maipo estuary in central Chile, B.LOOSE, Y. NIÑO C, C. ESCAURIAZA M.

- Two-dimensional simulation of a thermally stratified reservoir with high sediment-laden inflow, D.L. YOUNG, Q.H. LIN, K. MURUGESAN

- SPH simulation of solitary wave interaction with a curtaintype breakwater, SONGDONG SHAO

- High-resolution TVD schemes in finite volume method for hydraulic shock wave modelling, GWO-FONG LIN, JIHN-SUNG LAI, WEN-DAR GUO

- Two-dimensional and line jets in a weak cross-flow, J.F. HUANG, M.J. DAVIDSON, R.I. NOKES

- On incompressible turbulent flow: partial average based theory and applications, G. GAO and $Y$. YONG

- Vertically integrated numerical model to simulate breach formation by flow overtopping earth-filled dams: hydraulics, $R$. FRENETTE, A. MUNTEANU

- Discharge equations for venturimeter and orificemeter, PRABHATA K. SWAMEE

- Modified log-wake law for zero-pressure-gradient turbulent boundary layers, JUNKE GUO, PIERRE Y. JULIEN, ROBERT N. MERONEY 